\title{
A Practical Fit for the Critical Surface of NbTi
}

\author{
L. Botlura \\ CERN, LFC. Division, 1211 Geneva 23, Switzerland
}

\begin{abstract}
Known expressions for the critical temperature, critical held and Pinning foree in Nb'Ti are combined into a selfconsistent lit formula that provides the critical curtent density as a function of temperature and field. 'The main advantage of such a fit is the extended validity range. Data available ju literalure and measurements on LIIC strands are used to femonstrate the acenracy of the fit. The $J_{c}$ data-sets used to cover a range of field from $0 \mathrm{~T}$ to $9 \mathrm{~T}$ and temperature from $1.9 \mathrm{~K}$ to $9 \mathrm{~K}$. The standard devialion of the fits presented is of the order of $5 \%$ or better. This accuracy is generally sufficient for design purposes, extropolation and sealing of measured results. Better accuracy, e.g. for short sample limit prediction, ean be achieved restricting the domain of validity.
\end{abstract}

\section{INTRODUCTION}

Fitting formulate for the critical surface $J_{f}(B, T)$ of $\mathrm{NbTj}$ are commonly used for the engineering calculation of short sample limit, temperature and current margins, and for the calculation of magnetization. Several practical fits have been produced in the literature, see for example [1]-[4]. The main dawback of these fits is that often they have been tailoced to restricted ranges of field $B$ and temperature $T$, to a particular strand material, or are defined as complex piecewise polynomial fits, Surprisingly, the cousistent use of a Pinning tnodel and a temperature scaling law seems to have eluded common practice in magnet design. The purpose of this paper is to resort back to known expressions for the critical temperature, critical field and Pinning force density to produce a simple but general fit of the eritical surface of commercial NbTi. I will show by coniparison with published data that in spite of its simplicity this approach has a good engiteering value.

\section{CRITICAL SURFACE FiT}

Table I reporls the symbols used in the paper for the criticat parameters. It is useful to introduce the reduced temperature $t$ and the reduced field $b$ defined as follows:

$$
\begin{aligned}
& t=\frac{T}{T_{c \cdot 0}} \\
& b=\frac{B}{B_{* 2}(T)} .
\end{aligned}
$$

In accordance with the results of likin on Nb'i alloys [5], we neglect the dependence of all parameters on intrinsic

Mamusctipl fectivel Sepulmber 27, 1999 strain. The function chosen here for the fit of the critical surlace as a function of the reduced parameters $t$ and $b$ is given by:

$$
J_{c}=\frac{C_{0}}{B} b^{r}(1-b)^{n}\left(1-t^{n}\right)^{\gamma}
$$

Equation (3) has 4 free parameters: a normalization constant $C_{0}$, two parameters describing the dependence on the reduced field, $\alpha$ and $\beta$, and a parameter describing the dependence on the reduced temperature, $\gamma$. Note that $J_{r}$ in (3) depends on temperature explicitly through the last term and implicitly through the critical field dependence which enters the definition of the reduced field. $\Lambda$ suitable critical lield dependence on temperature can been taken from Lubell [1]:

$$
B_{c 2}=B_{r 20}\left(1-t^{n}\right)
$$

where the exponent $n=1.7$ appears to provide a satisfactory fit to most alloy compositions. Hor completeness, we recall that the critical temperature dependence on field is obtained as the inverse of the critical field relation (4);

$$
T_{c}=T_{c 0}\left(1-\frac{D}{B_{t: 20}}\right)^{\frac{1}{n}}
$$

The final parameter of interest for practical use is the current sharing temperature $T_{r i i}$. This is defined implicitly as the remperature at which the operating current density in NbTi $f_{o r}$ equals the critical current density, or:

$$
J_{w p}=\frac{C_{0}}{B} b^{\alpha}(1-b)^{a}\left(1-t_{r y}^{n}\right)^{\gamma}
$$

\begin{tabular}{|c|c|c|}
\hline$J_{n}(B, T)$ & {$\left[\mathrm{A} / \mathrm{m}^{2}\right]$} & cribial cument density \\
\hline$T_{c}(B)$ & {$[\mathrm{K}]$} & critionl temperature \\
\hline$n_{t 2}\left(1^{1}\right)$ & {$[1]$} & upper critienl field \\
\hline $\mathrm{T}_{\mathrm{c} d}$ & K $]$ & maximm critionl temperature (at $\mathrm{B}=0$ ) \\
\hline$b_{\mathrm{r} 20}$ & {$[\mathrm{~T}]$} & maximum upper critical lick (at $\mathrm{T}=0$ ) \\
\hline$F_{\mathrm{\mu}}(B, T)$ & {$\left[\mathrm{N} / \mathrm{m}^{3}\right]$} & Pinniug force density \\
\hline$\gamma_{r-1}\left(\beta, f_{p, n}\right)$ & {$[\mathbf{K}]$} & cunent sliaring temperature \\
\hline
\end{tabular}

where $t_{c s}$ is the reduced current sharing temperature:

$$
t_{c s}=\frac{T_{c s}}{T_{c 0}}
$$

'IAIJLE I

CRITKAL FUNCTIONS AND PABAMETJIRS 
Equations (6) and (7) can be solved numerically (e g. by an iterative bisection nethod), providing an arbitrarily aceurate value of the current sharing temperature.

Equation (3) has a well-established structure that can be derived is a direct consequence of the temperature scaling law of Fietz and Webb [6]-[7]. Using the definition of the crilical field given by (4) we can rearrange the terms in (3) and express the Pinning force density $F_{p}=J_{c} \times B$ in the following manner:

$$
F_{p}=J_{r:} \times B=f(b) U_{r 2}^{\gamma}
$$

where $f(b)$ is the Piming curve of the material $[7]$ that depends on the reduced licid only, and the temperature scaling through $B_{r^{2}}$ is indeed in iccordance with [G]. By comparison of (3) and (8) we see that the Pinning curve for the material $f(b)$ assumed in the lit is given by:

$$
f(b)=\frac{C_{0}}{B_{c 2 !}^{\gamma}} \cdot b^{\alpha}(1-b)^{p}
$$

It is genurally more usclul to define a normalized Pinning curve:

$$
\frac{f(b)}{f_{m: 1 *}} \propto b^{a}(1-b)^{\prime}
$$

that exhibits a maximum at $b_{\operatorname{mix}}=\alpha(\alpha+\beta)[8]$. The above functional dependence comes from standard practice in the analysis of P'inning data from $J_{c}$ mensurements [5]-17]-[8]. Equalion (10) shows that the two exponents $\alpha$ and $\beta$ are solely related to the dependence of the normalized Piming force on the reduced field, while the exponent $\gamma$ is determined by the temperature scaling law as expressed by (8).

\section{AP'JIICATIONS TO PUIBISHET JC DATA SET'S}

The validity of the fit can only be confirmed by direct comparison with measurements. To demonstrate the accuracy achicvable with the formulae given above we have fitted data sets of NbTi $J_{r}$ data. Most of these data are the same as collected by Green in Ret. [3]. In particulat we have chosen in Ra[. [3] the data from Spencer [9], Somerkoski [10] and T'aylor [11]. In addition the data set used by Morgan [4] and Hudson [12] have been tested. They refer to different strands, produced during a lime span of about 10 years, with different alloy composition and very wide varialion of values for $J_{*}$. In all cases the value of the critical current density $J_{t}$ has becn normalized to the moasurement at $5 \mathrm{~T}$ and $4.2 \mathrm{~K}$ for convenience of representation.

The data set of Spencer [9] refers to a Nb-46.5Ti alloy tested at fields in the range 0 ' $\mathrm{T}$ to $8 \mathrm{~T}$ and temperatures in the range $4.2 \mathrm{~K}$ to $9 \mathrm{~K}$. $\Lambda$ total of 33 measured points have been fitted, with the results shown in lig. 1. The maximum local error, relative to the reference value of $J_{c}$, at $4.2 \mathrm{~K}, 5 \mathrm{~T}$, is

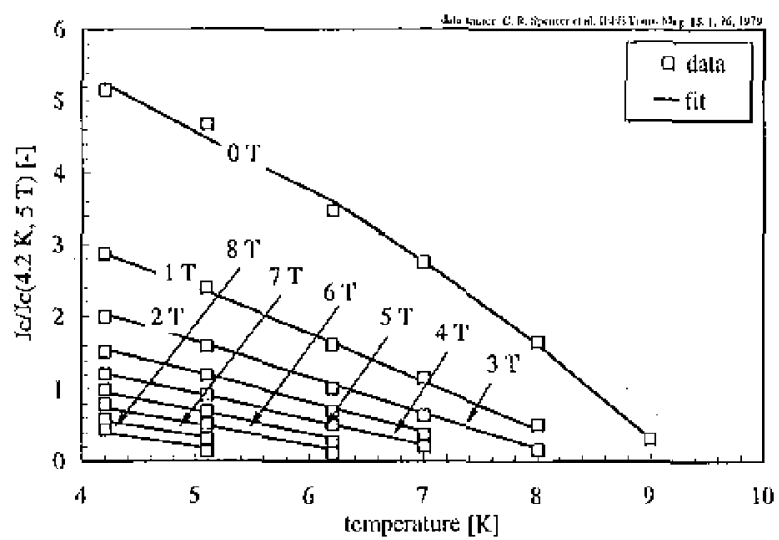

Hig. 1. Temperature dependence or the nortnalized $J_{c}(B, T) / J_{s}(57,4.2 \mathrm{~K})$ as reporled by spencer $\mid 91$ on $\mathrm{Nb}-46.5 \mathrm{Ti}$ wire, and fitted using (3). Ficld values are inclicsted on the plot.

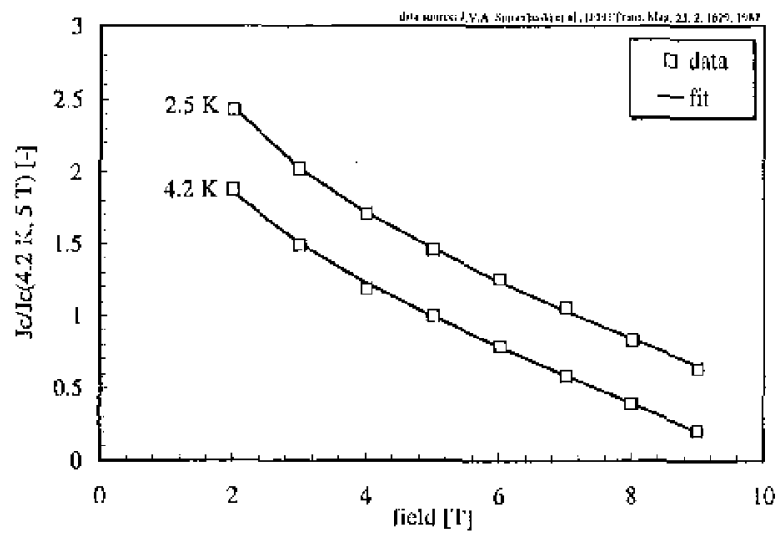

Fig. 2. Field denendence of the normalized $J_{r}(I t, T) / I_{c}(5 \mathrm{~T}, 4.2 \mathrm{~K})$ as reported by somerkoski l]0] on Nb-46.5Ti wire, and fitted using $(3)$. Temperature vilues ate inflicated on the plof.

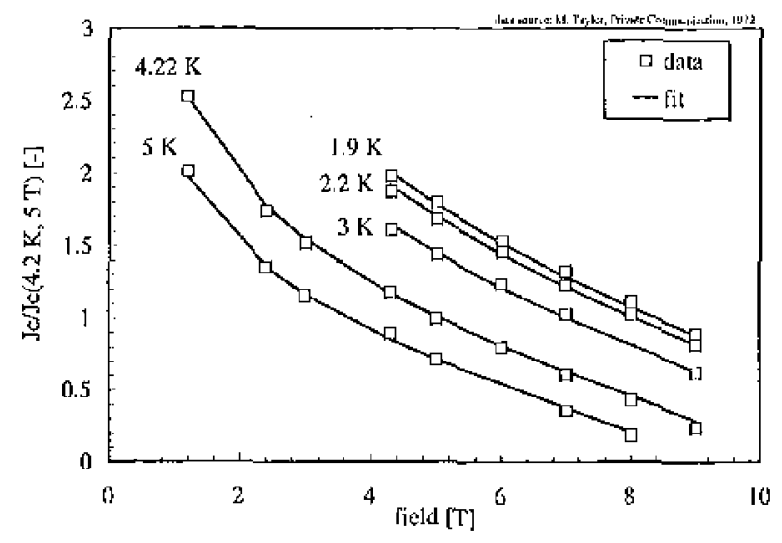

Pig. 3. Lield dependence of the normalized $J_{c}(B, T) f J_{c}(S \mathrm{~T}, 4,2 \mathrm{~K})$ as reporteal by Taylor [11] on Nb-44Ti wire, and fitted using (3). Telnjeraluse values are indieated on the plot. 
approximately $20 \%$ and is found at zero background field. The overall standard deviation of the fit is about $5 \%$ (again referred to the reference value of $J_{f}(4.2 \mathrm{~K}, 5 \mathrm{~T})$ ).

The second data set, collecling data from Somerkoski [10] also refers to a Nb-46.5Ti alloy tested at fields in the range of 2 to $9 \mathrm{~T}$ and temperatures of 2.5 and $4.2 \mathrm{~K}$. The data set consists of 16 points. The result of the fit and the fit parameters used are reported in Fig. 2. The maxinum local errot achieved is of the order of $3.5 \%$ while the standard deviation is about $1.5 \%$.

The third data set is from 'Iaylor [11], for a Nb-44ר'i alloy tested at ficlds in the range of 1 to 9 ' $\mathrm{l}$ ' and temperatures of $1.9,2.2,3,4.22$ and $5 \mathrm{~K}$. The data set consists of 35 points. The results are reported in [Fig. 3. The maximum local error achieved is of the order of $3.5 \%$ and the standard deviation is :tbout $1.9 \%$.

The data of Morgan [4] for SSC NbTi wire consists of 44 points in the ficld range of $4108 \mathrm{~T}$ and temperature range of 2.5 to $7.5 \mathrm{~K}$. Results on the fit are presented in Fig. 4.

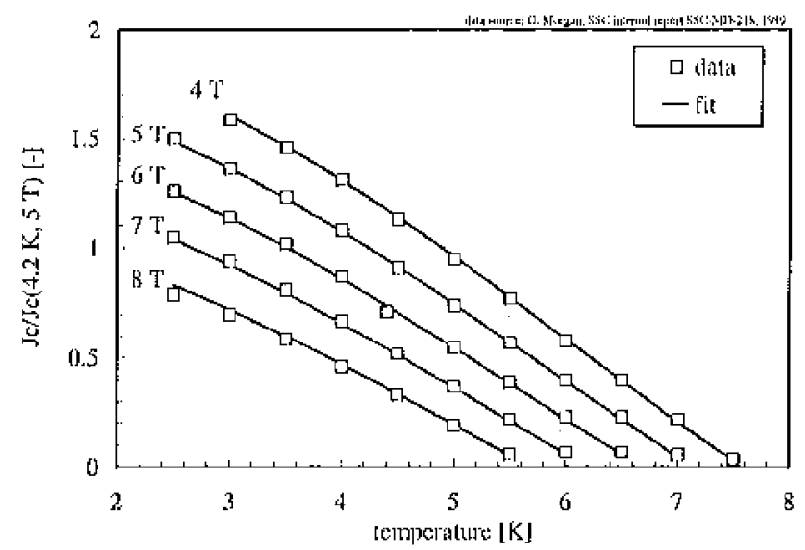

Fig. 4. Temperature dependenee of the nommalized $S_{c}(B, T) / J_{r}(5 \mathrm{~T}, 4.2 \mathrm{~K})$ as lepotted by Morgan [a] oft SSC NbTi wite, and [illed using (3), Field values are indienter on the plot.

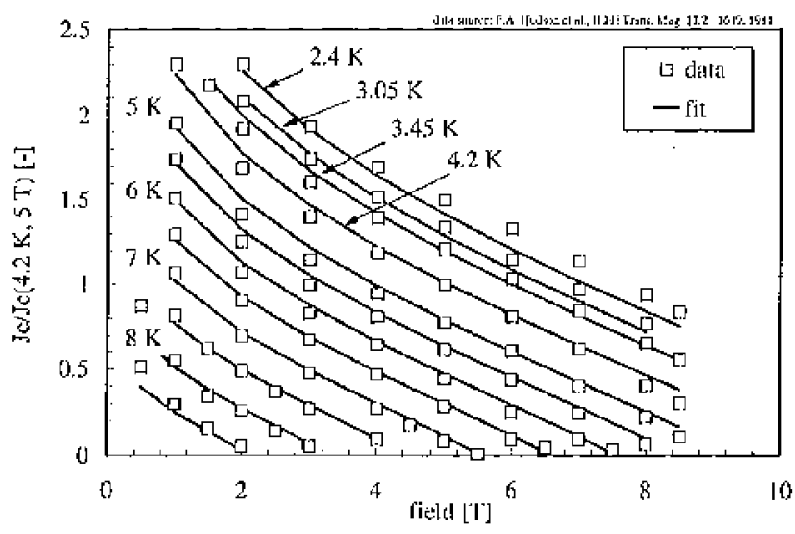

Fig. 5. Ficht depentence of the nomalized $J_{t}(J, J) / J_{t}(5 \mathrm{I}, 4.2 \mathrm{~K})$ as reported by Iudson [ 12 I on Nb-46.5T' [M] wires, and tilted using (3). "Tempenalute values as indiented on the plot. In the interval 5 10 $8.5 \mathrm{~K}$ the spacing of the curves is $0.5 \mathrm{~K}$.
TABI.F IL

SUMMARY Ol RESULTS IOR THL TLST ITTS MGAINST PLIBLISIIED JCDATA SHIS (IDATA SOURCE IN RTAITRENCLS QUOTLD)

\begin{tabular}{|c|c|c|c|c|c|c|}
\hline sontree & & Rel.[9] & Ref. [10] & Ref.|11] & Ref.[A] & Rol' [12] \\
\hline Ii content & $1 \%]$ & 46.5 & 46.5 & 44 & nat & 46,5 \\
\hline points & {$[-]$} & 33 & 16 & 35 & 44 & 88 \\
\hline$B_{020}$ & [T] & 14.5 & 14.2 & .14 .4 & 14.4 & 14.25 \\
\hline$\eta_{n}$ & [K] & 9.2 & 8.5 & 8.9 & 9,2 & 9.35 \\
\hline$C_{i}$ & ["T] & 23.8 & 28.6 & 28.5 & 37.7 & 28.4 \\
\hline$a$ & {$[-]$} & 0.57 & 0.76 & 0.64 & 0.89 & 0.80 \\
\hline$\theta$ & $1-1$ & 0.90 & 0.85 & 0.75 & 1.10 & 0.39 \\
\hline$\gamma$ & {$[-]$} & 1.90 & 1.76 & 2.30 & 2.09 & 1.87 \\
\hline$a_{\mathrm{k} / L i}$ & {$[\%]$} & 20 & 3,5 & 4.1 & 1.5 & 17.1 \\
\hline$\sigma$ & [\%] & 5 & 1.5 & 2.3 & 1.2 & 50 \\
\hline
\end{tabular}

Maximum local error is below $1.5 \%$, and standard deviation is $1.2 \%$

Finally, the data of I-Tudson [12] refers to Nb-46.5Ti wire produced by JMI, and consists of 88 points in the field range of 0.5 to $9 \mathrm{~T}$ and temperature range of 2.5 to $8.5 \mathrm{~K}$. This is the data sel with the largest ficld and temperature oxtension tested. Restlits of the [jt are presented in litg. 5. Maximum local error is $17 \%$ (again at very low ficld, below $\mathrm{l} T$, and high temperature, above $8 \mathrm{~K}$ ). At low temperature $(2.4 \mathrm{~K}$ ) and high field (above 6 to $8 \mathrm{~T}$ ) the local error ranges from 8 to $12 \%$. The overall standard deviation of the fit is $5 \%$.

Table II contains a summary of the number of points used for each dala set, the fit parameters, the maximum local ertor $\varepsilon_{n i x}$ and the slandard deviation $\sigma$. Note that the value quoted for $C_{o}$ relers to the fits of normalized $J_{0}$, and has therefore urits of $[T]$, instcad of $\left[\mathrm{A} T / \mathrm{m}^{2}\right]$ as can be derived from (3). The lypical standard deviation for the complete data set is always in the range of 2 to $5 \%$, with maximum errors in the range of $5 \%$. Higher errors, of the order of $20 \%$, should be expected al the boundary of the fitting range, especially at low field. Comparing the values of the fitting parnmeters $\alpha, \beta$ and $\gamma$ we see that their lange of variation is restricted. In particular $\alpha$ is of the order of 0.5 to 0.8 , implying an approximate $B^{-1 / 2}$ dependence for $J_{c}$ at low field, as indeed found from $J_{c}$ and magnetization measurements [2]. The second parameter, $\beta$, has values close to 1 . linally, the third parameter, $\gamma$, is in the range of 2 .

The values of $B_{\text {r:2o }}$ and $T_{\text {rot }}$ were not optimized in the fits. Measured or quoted data for the material were used if available in the references. For the cuses where no values were guoted the choice was restricted in order to remain consistent with the typical material characteristics reported in the literature ( $B_{i 20}$ around 14 to $14.5 \mathrm{~T}$ and $T_{i 0}$ around $9 \mathrm{~K}$ ). Botter tming of the fits would be possible considering also these parameters as free, and adjusling them gencrally to lower values than those taken above. In this case howeve the physical meaning of the upper eritical field and temperalure would be lost, and therefore this way was not pursued further.

Using (9) it is possible to compute the normalized pinning curve for cach dala set litted. The curves obtained are reporled in Fig. 6, and are compared to data reported by Larbalestier [7] representing the typical behaviour of a $\mathrm{Nb}-46.5 \mathrm{Ti}$ alloy. Note that this data can be filled using (9) with a choice of $\alpha=0.65$ and $\beta=1$. $\Lambda$ s showl in Fig. 6 the 


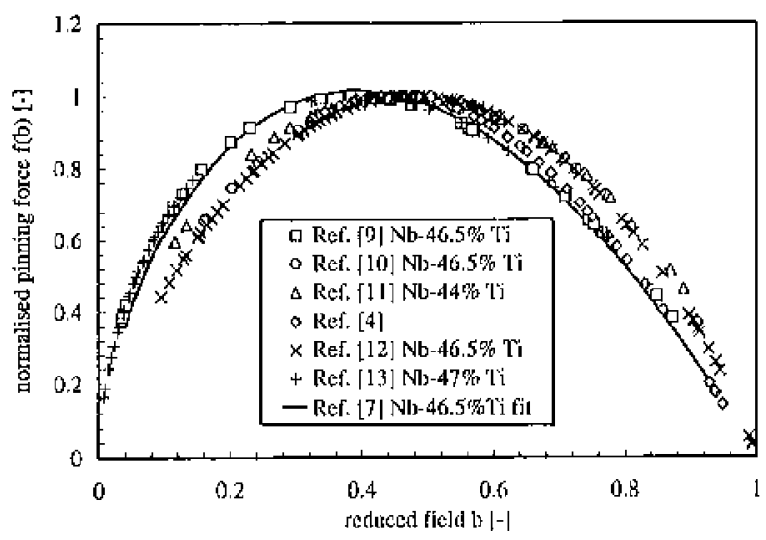

Fig. 6. Normalized Pinning force as computed from the critical eurrent fits reported here. The values are compared to a that fit ciluoled in Rel', [7].

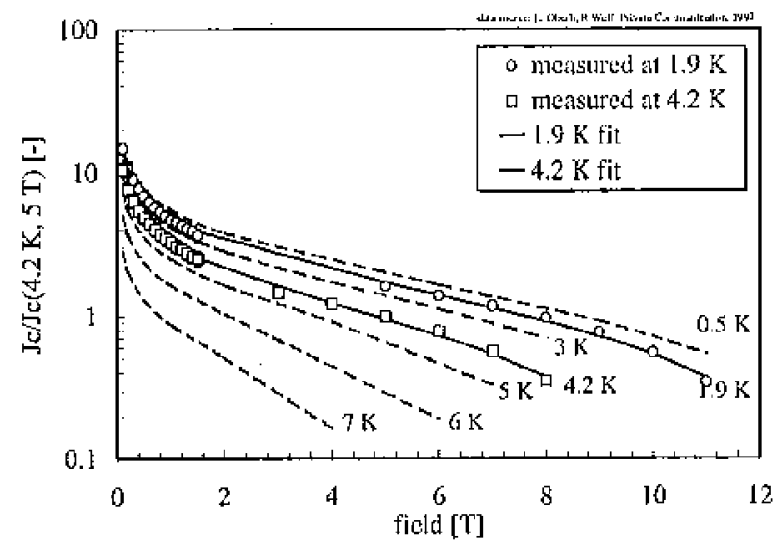

Fig. 7 . Field dependence of the nermalized $J_{c}(\beta, h) / J_{c}(5 \mathrm{~T}, 4.2 \mathrm{~K})$ lor a lypical LHC strand [1.3]-[1 4 , nensured at 1.9 and $4.2 \mathrm{~K}$. The fit to the data is shown (solid lines) togelher with curies generated for clillerent icmpertures (dashed lines).

Pinning curves obtained are in reasonable agreement with the expected behaviout. We recall that the shape of the Pinning curve is alloy and precess specific, and is not a material characteristic. Therefore variations of the Piming curve must be expected among different strands.

\section{EXAMPIE OF JC SCALING FOR AN LIIC STRAND}

An example of practical application of the fitting formula is shown in Fig. 7. The $I_{t}$ data at $1.9 \mathrm{~K}$ and $4.2 \mathrm{~K}$ is relative to a typical $\mathrm{Nb}-47 \mathrm{Ti}$ strand for the cable of the outer lnyer of an L.HC main bending dipole. Data at high field (above $3 \mathrm{~T}$ ) are derived from $l_{t}$, measurements [13], while the data at low field (below 1.5 'l') are deduced from magnetization measurcments on the same strand [14]. The crro: on the data at low lictd can be large (of the order of $10 \%$ ) because of the uncertainty on the details of filament magnetization.

The fit was obtained taking $B_{c 20}=14.5 \mathrm{~T}$ and $7_{c 0}=9.2 \mathrm{~K}$ The lit parameters are $C_{0}=27.04 \mathrm{~T}, \alpha=0.57, \beta=0.9$ and $y=2.32$. The standard deviation of the fit is approximately $5 \%$. 'lthe maximum local error (at low field) is $11 \%$, while at high ficld the local error is of the order of 1 to $5 \%$. The Pinning curve for the strand material is reported in Fig. 6 and shows an excellent agreement with the expected behaviour of the alloy. In addition to the fitted data Fig. 7 shows the $d_{r}$ curves generated for different lemperalures.

\section{CONCLUSIONS}

The use of a simple but consistent Pinning model and temperature scaling for the critical surlace of NbTi appears to provide a satisfinctory match to several $J_{\text {c }}$ data sets on different strands. The resulting fit is valid over the full range of Field and temperature. Typical overall litting accuracy is of the order of $5 \%$ and better, with maximum local errors of the order of $20 \%$ (mostly in the low field region, below $1 \mathrm{~T}$ ). These values are adequate fot comparisons among strands, correlations, projection of performance and calculation of magnetization at low ficld.

\section{ACKNOWL.IBGMIN'}

I gratefully acknowledge the collaboration of L.Oberli and $\mathrm{R}$. Wolf (CERN) who have provided the $\mathrm{rc}$ and magnetization measurement data on the LIIC strand.

\section{RIIFLRLNCES}

[1] M.S. lubell, "Etmpirical scaling formulas for critical current alud ericical rield For commencial NiTi," WEE Troms Mas., vol. 19 (3), ค. 754,1983 .

[2] M.A. Green, "Generaten of the Jc, IEc, Te surlace for commercial suterconductors using reduced-state psitameters," I.BL and S.SC Intemal Repott, SSC-N 502 , LI3L-24875, 1988.

[3] MA Green, "Calculiting olse Je, B, T surlace lor miobium titanium using a reduced-siate modkl," IEEE Trour. Mag., vol. 25 (2), p. 2199, 1989.

[4] G. Morgall "A comparison of (wo analytic forms for the le (1),T")

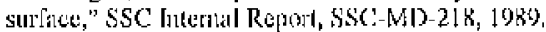

[5] J.W. Ekick, "Simain sealing law for flux pinuing in NoTi, Nb3Sm,

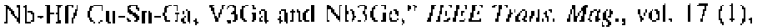
एр. $658-661,1081$.

[6] W.A. Few, W,W. Wobl, "Hysteres in superconducting alloys temperatute and field dependence of dislocation Pinning if niobium alloys," Plyw. Mew, vol. 178 (2), ,1]. 657-667, 1969.

[7] D. Litbalestice, NbTi alloy suderconductors - prosent stalus and polerial for improvement," Adv. Cryo.long., vol. 26, pp. 10-36, 1980

[8] F.J Kramer, "Scaling laws for flux p'imning in tard stpercondictors," J. Appl. 1'iys., vol. 44 (3), [1. 1360-1370, 1073.

[9] C. R, Spencer ct al, 'the temperature and magnetic lield dependence of superconducting eritical enrent densities of mulifilanentary Nb3Sn and NbTi composite wires," IElKe Trans. Merg, vol, 15 (1), p. 76, 1979.

[10] J.Y.A. Somerkoski et al., Structure and supercoulucting property

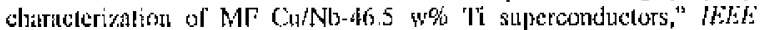
Trash. Mag, val. 23 (2), [1. 1629, 1987?

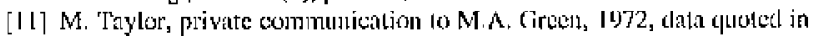
Ref. [3.

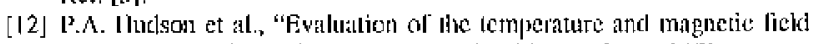
dependenes of critical current demsilies of Irultifilamentaty

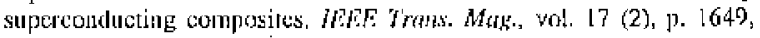
1981.

[13] I., Oberli, CRRN, Geneva, Switzerland, private comnumieation, I096.

[14] S. I. E Naotr, el al. "Magnetization measurements on LliC superconducting strands," IEFE Than. Appl. Sip., vol. 9 (2), p). 176.7-1766, 1999 . 